\title{
Symmetric spatio-temporal focusing of ultrashort pulses
}

\author{
A. Treffer ${ }^{1}$, D. Kühn ${ }^{2}$, F. Wyrowski ${ }^{2}$, R. Grunwald ${ }^{1}$ \\ ${ }^{1}$ Max Born Institute for Nonlinear Optics and Short-Pulse Spectroscopy, \\ 12489 Berlin, Germany \\ ${ }^{2}$ Institute of Applied Physics, Friedrich-Schiller University, Albert-Einstein-Straße 15, \\ 07745 Jena, Germany \\ email: treffer@mbi-berlin.de
}

\begin{abstract}
Summary
Simultaneous spatio-temporal focusing of few-cycle pulses is usually performed by stretcher-compressor configurations causing pulse-front tilt. By shaping and superimposing two reciprocal sub-beams, opposite tilts can compensate each other. Pulse propagation for asymmetric and symmetric focusing of near infrared 5-fspulses is studied by numerical simulations and proof-of-principle experiments.
\end{abstract}

\section{Introduction}

The propagation of ultrashort pulses in optical media is often limited by nonlinear effects and damage because of high peak intensities. These constraints can be circumvented by stretching and compressing pulses by dispersive components. A particular stretcher-compressor concept is known as simultaneous spatio-temporal focusing (SSTF) [1-2]. The main goal of SSTF is to provide a focus with minimum pulse duration at the position of interest. Theoretical studies demonstrated the capability of the approach [3]. Simultaneous localization in space and time, along with robust temporal transfer, enables to investigate remote nonlinear processes behind dispersive elements even with low-pulse-energy Ti:sapphire oscillators. Therefore, SSTF is of increasing interest for applications in materials processing [4], microscopy [5], plasma physics [6], or nonlinear optics. Previously, a single-channel SSTF system was studied by numerical simulations [3]. It was shown that dispersion causes travel time differences that lead to pulse-front tilt (PFT) with characteristic signatures in spatial, spectral and temporal domain. Here we report on an alternative approach based on two SSTF channels with reciprocal grating configuration that allows for compensating PFT and thus to form symmetric pulse fronts. Results of numerical studies as well as first proof-of-principle experiments are presented.

\section{Symmetric stretcher-compressor approach}

The schematic drawings in Fig. 1a and Fig. 1b show a conventional single-path twograting setup for SSTF and a two-path system, respectively. The reciprocal grating arrangement (Fig. 1b) is capable to symmetrize the focus and thus to partially compensate for dispersion-induced pulse-front tilt (PFT). If the length of both channels is properly adjusted, the phase difference in the center is minimized. The degree of compensation of PFT depends on the superposition angle and the temporal coherence of the pulse. Physical optics simulations with VirtualLab Fusion software were used to calculate the pulse-front tilt of the traditional and the two-path system. Figs. 2a-f show the field amplitude at the detector plane of the system depicted in Fig. 1a, the same system mirrored at its symmetry axis, and the superposition of both, respectively (Fig 1b) with and without dispersion. Predictions were confirmed by proof-of-principle experiments with a Ti:sapphire laser oscillator 
(Laser Quantum, central wavelength $700 \mathrm{~nm}$, minimum pulse duration $9 \mathrm{fs}$, prechirped) stretched by a prism pair $(1200 \mathrm{l} / \mathrm{mm})$ and lens focusing $(\mathrm{f}=75.6 \mathrm{~mm})$. Pulse characterization was performed with a GRENOUILLE setup. Currently, experimental conditions are confined to reduce parasitic chirp effects.
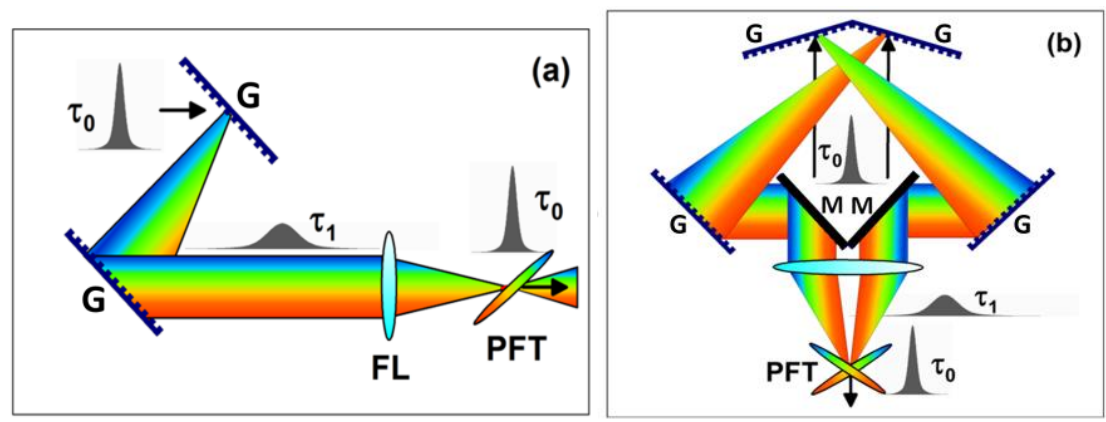

Fig. 1. Configurations for spatio-temporal focusing: (a) double-grating setup with asymmetric focusing, (b) 4-grating setup with symmetric focusing (PFT = pulse-front tilt, $G=$ grating, FL = ocusing lens, $\tau_{0}, \tau_{1}=$ pulse durations with and without compression, $M=$ mirror, schematically).
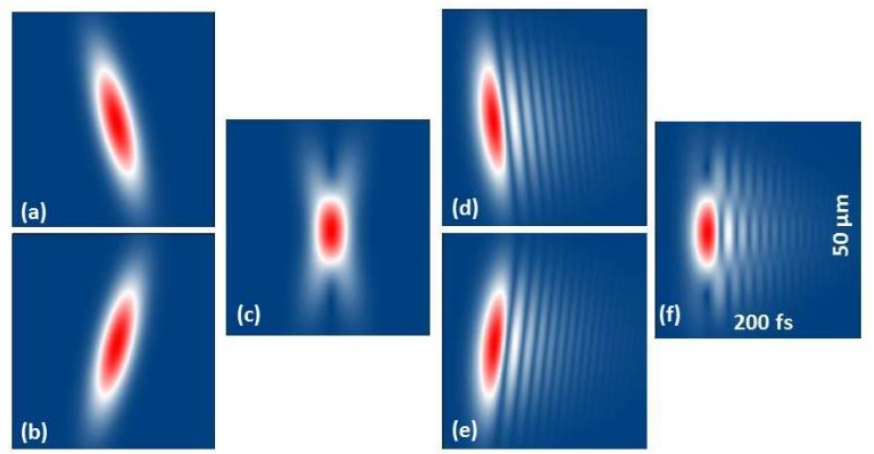

Fig. 2. Field distribution of single and dual channel configuration in the focal region simulated for a Gaussian pulse (center wavelength $619 \mathrm{~nm}$, FWHM bandwidth $40 \mathrm{~nm}$, initial FWHM pulse duration $14.2 \mathrm{fs}, \mathrm{GDD}=9600 \mathrm{fs} 2$ ); perfect compensation: (a), (b) left and right arm, (c) superposition of both; with realistic chirp: (d), (e), (f), respectively (normalized). The grating orientation determines the direction of pulse front tilt. Therefore, the superposition creates a cross-shaped field (see $c, f)$.

\section{Conclusions}

A double-stretcher-compressor scheme for simultaneous spatio-temporal focusing was proposed to symmetrize the pulse front. Proof-of-principle experiments confirm the approach. To enable a tuning of the superposition angle, adaptive configurations on the basis of MEMS components will be studied in a next step.

The work was supported by DFG (Gr 1782/16-1).

\section{References}

[1] G. Zhu, J. v. Howe, M. Durst et al., Opt. Express 13, 2153-2159 (2005)

[2] D. Oron, E. Tal, and Y. Silberberg, Opt. Express 13, 1468-1476 (2005)

[3] S. Zhang, F. Wyrowski, R. Kammel and S. Nolte, Proc. SPIE 8972, 89721B (2014)

[4] B. Sun, P. S. Salter, C. Roider et al., Light: Sci. \& Appl. 7, 17117 (2018)

[5] G. Song, A. Nakamura, K. Hirosawa et al., Rev. Sci. Instrum. 86, 083701 (2015)

[6] Z. Wang, B. Teng, G. Li et al., Opt. Lett. 40, 5726-5729 (2015) 